\title{
SIMPLE BROADRANGE TUNING OF FIBRE-DFB LASERS
}

\author{
S.Y. Set (1), M. Ibsen (2)*, C.S. Goh (3) and K. Kikuchi (3)
}

(1) Micron Optics Inc., 1900 Century Place, Suite 200, Atlanta, GA 30345, USA.

(2) ORC - University of Southampton, University Road, Southampton SOl 7 1 BJ, UK.

Also with Southampton Photonics Ltd., Chilworth Science Centre, Southampton SO 16 7NS, UK.

(3) Research Center for Advanced Science and Technology, University of Tokyo

Optical Devices Laboratory, 4-6-1 Komaba, Meguroku, Tokyo 153-8904, Japan.

All-fibre distributed-feedback (DFB) lasers providing continuous tuning over $27 \mathrm{~nm}$ are demonstrated for the first time. Extended wavelength coverage of the lasers is obtained using a simple bend-tuning technique which delivers undistorted outputs over the full tuning-range.

\section{Introduction}

Fibre grating distributed-feedback (DFB) lasers exhibit many attractive features for application as laser sources in optical communication / 1/. For example, their advantages over semiconductor DFB lasers include ease of fabrication, inherent fibre compatibility for enhanced transparency, high output power, $\sim 10 \mathrm{x}$ lower thermal sensitivity and superior optical signal-to-noise ratio. Previously, fibre DFB lasers fabricated in $5 \mathrm{~cm}$-long Er/Yb co-doped fibres have been demonstrated with single polarisation and output power in excess of $13 \mathrm{dBm}$, optical signal-to-noise ratios (SNR) of $>50 \mathrm{~dB}(0.1 \mathrm{~nm}$ resolution), and line-widths of $\sim 10 \mathrm{kHz} / 2 /$. Additionally, they have been demonstrated to be highly suitable for application as transmitter sources in wavelength division multiplexed transmission systems $/ 3 /$.

As WDM optical communication systems evolve from simple point-to-point links to dynamic optical networks, wavelength tunability of the devices in these systems will become necessary. In order to accommodate these requirements, a number of techniques to tune the operational wavelength of $\mathrm{CW}$ fibre lasers have been proposed. One technique for wavelength-tuning a fibre DFB laser was by mechanically stretching the device, as shown in $/ 4 /$ for a $3 \mathrm{~nm}$ tuning. The maximum tuning-range using this method is limited by the breaking tensile strain of silica fibre, which is typically $\sim 1 \%$, corresponding to $12 \mathrm{~nm}$ of wavelength shift. Secondly, Ball et al. 15/ demonstrated continuous tuning over a $32 \mathrm{~nm}$ wavelength range by compression tuning a fibre distributed Bragg reflector (DBR) laser in a master-oscillator/power-amplifier (MOPA) conf iguration.

However, this system was complicated by the requirement to maintain the straightness of the fibre to prevent buckling using complex ferrule design. Besides, compression was achieved by using a stepper-motor, which is bulky and relatively slow. Moreover, the MOPA configuration could potentially sacrifice the optical SNR. Pan et al. $16 /$ demonstrated a third technique by sandwiching a hybrid DFB/DBR laser between two high-thermal expansion Teflon plates. This system is somewhat complex, and requires a temperature range of $80^{\circ} \mathrm{C}$ to achieve the $11 \mathrm{~nm}$ tuning-range.

In this paper, we demonstrate simple wavelength tuming of all-fibre DFB lasers over $27 \mathrm{~nm}$. The wavelength tuning method applied is based on a bend-tuning technique that we have demonstrated recently for tuning a passive fibre Bragg grating filters over $40 \mathrm{~nm} / 7 /$. Our results represent the broadest wavelength tuning-range achieved, to the best of our mowledge, for any DFB laser configuration including semiconductor DFB lasers.

\section{Device Design}

The devices demonstrated here are $4 \mathrm{~cm}$ and $5 \mathrm{~cm}$ in length, and are written into a highly doped $\mathrm{Er} / \mathrm{Yb} / \mathrm{P} / \mathrm{Al} / \mathrm{Si}$ fibre with an annular photosensitive $\mathrm{B} / \mathrm{Ge} / \mathrm{Si}$ region to the core $/ 8 /$ using a fabrication system operating at $244 \mathrm{~nm}$. Previously these lasers have been demonstrated to operate at power levels of up to $14 \mathrm{dBm}$ when pumped with powers in excess of $20 \mathrm{dBm}$ at $976 \mathrm{~nm}$. To obtain unidirectional output, the fibre lasers are written with a discrete $\pi$ phase-shift in a $6 \%$ off-centre position $/ 2 /$ resulting in an output power ratio of $\sim 50$ : 1 . Furthermore, these devices operate with singlepolarisation outputs. After fabrication, the fibre-lasers are cleaved and spliced onto standard fibre at both ends. Otherwise, the high absorption in the excess Er/Yb fibre would prevent sufficient pump-power in reaching the DFB active region.

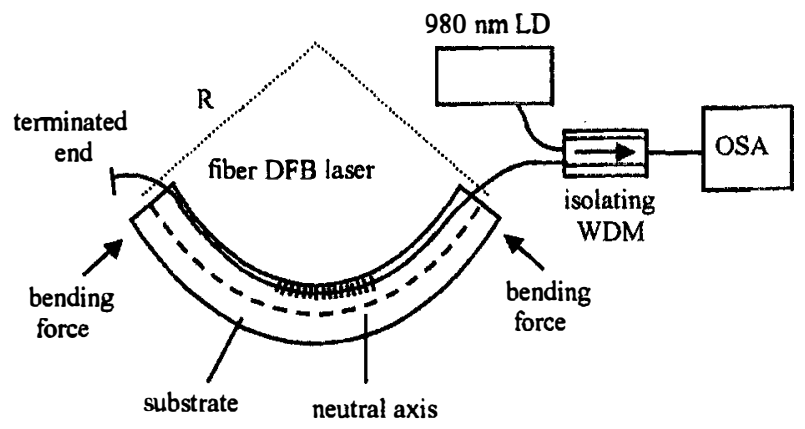

Figure 1: Design schematic of the tunable fibre DFB laser configuration. 
Then the fibre DFB lasers are mounted in an of $\sim$ neutral axial position on a bendable substrate (Fig. I), which allows tuning of the devices by both traction andcompression/7/. The wavelength shift $\Delta \lambda$ using this technique can then be estimated by:

$$
\Delta \lambda=0.78 \varepsilon \lambda, \text { with } \varepsilon=d / R
$$

where $\lambda$ is the center wavelength of the device at idle state, $\varepsilon$ is the applied strain/stress, $R$ is the bending arc-radius and $d$ is the off-neutral-axis displacement.

In our design, a relatively large displacement factor, $d$, of $1.6 \mathrm{~mm}$ enables wide wavelength tunability with little constraint in the bending radius. A $20 \mathrm{~nm}$ wavelength shift will require only a bending radius of $\sim 10 \mathrm{~cm}$.

\section{Experiment, Results and Discussion}

The fibre DFB lasers are backward pumped by a $976 \mathrm{~nm}$ laser diode through an isolating wavelength coupler at $60 \mathrm{~mW}$. The output spectra of the lasers are monitored using an optical spectrum analyser. Fig. 2 shows the output spectra of the $4 \mathrm{~cm}$ device which was tuned over $\sim 27 \mathrm{~nm}$ from $1524 \mathrm{~nm}$ to $1551 \mathrm{~nm}$. The normal (relaxed) operating wavelength of the laser was $1546.5 \mathrm{~nm}$ with an output power around $0 \mathrm{dBm}$. To demonstrate the tuning technique, we have tuned the laser by $4.5 \mathrm{~nm}$ or $\sim 0.4 \%$ in extension mode and $22.5 \mathrm{~nm}$ or $\sim 1.9 \%$ in compression mode. The output power of the laser follows the Er/Yb gain-profile as expected. At the lower wavelength region, some excess loss may be due to the slight bend loss. The sharp drop in power at $1524 \mathrm{~nm}$ is due to the $\mathrm{Er} / \mathrm{Yb}$ gain roll-off, below which the gain is too low for laser operation. Note that we have not seen any bend-tuning limitation, and it would be possible to tune the laser further down in wavelength if the gain was sufficient. To demonstrate higher power operation, we successfully wavelength tuned the $5 \mathrm{~cm}$-long fibre DFB laser over $22 \mathrm{~nm}$. This laser offers a higher output power of up to $9 \mathrm{dBm}$ due to the longer length as shown in Fig.3. In this case we limited the tuning range to the highest gain region, from $1534 \mathrm{~nm}-1556 \mathrm{~nm}$, to show a near uniform output power operation. In order to maintain a high laser output power throughout the tuning-range, the bending uniformity had to be maintained.

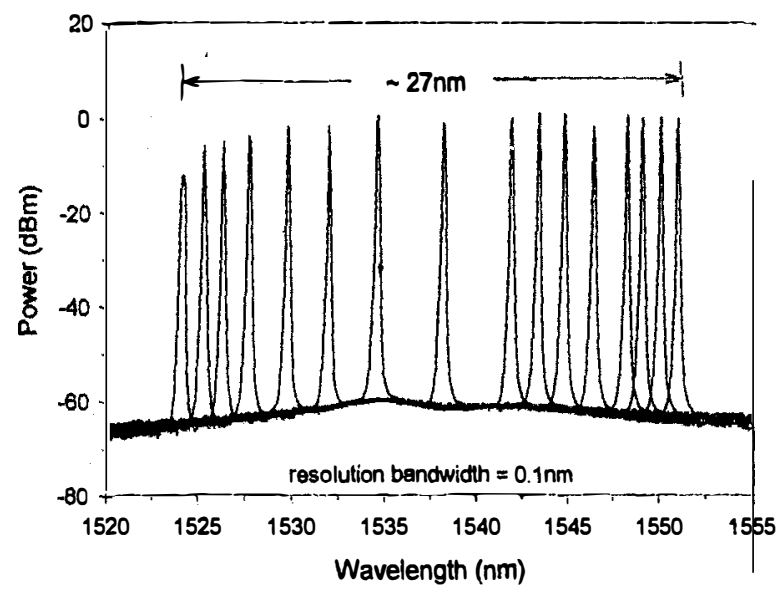

Figure 2: Output spectra of the $4 \mathrm{~cm}$-long fibre DFB laser wavelength tuned over $27 \mathrm{~nm}$.

Currently, the tuning range of the devices is mainly limitedby the strength of the splice points. Further improvements to the tuning-range should be possible with proper design . of a uniform bending mechanism and a technique to prevent stresses across the splice points. Despite this limitation, high quality outputs are maintained across the full range as demonstrated in Fig 2 and Fig 3 .

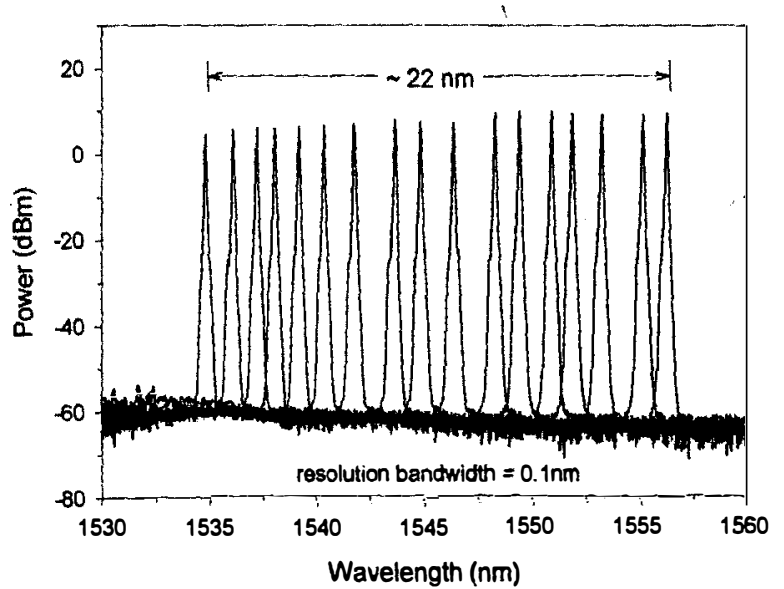

Figure 3: Output spectra of the $5 \mathrm{~cm}$-long high power fibre DFB laser tuned over $22 \mathrm{~nm}$. 
The bending substrate also serves as a good heat-sink base to cool the fibre DFB lasers. Due to the short length and high absorption of the lasers, significant heating occurs $/ 1 /$ which can distort the stability of the output power. If the laser is not heat-sinked properly, it would give a reduced output power and an unstable polarization operation. Continuous modehop firee tuning of lasers requires strict control of all the parameters in the cavity. Because the firequency of the lasers are dictated by the Bragg grating, unifiorm tuning of this is necessary if the overall laser perfiormance is to be maintained. However, despite the requirements fior bending unifiormity, the output power can easily be controlled by introducing intentional slight non-unifiorm bend to compensate fior gain variation across the wavelength window, without adjustment of the pump-power to the lasers.

\section{Conclusion}

We have demonstrated all-fiibre DFB lasers, which ofifier continuous wavelength tunability up to $27 \mathrm{~nm}$ using a simple bend tuning technique, which allows both and axial strain and compression. The devices remain operating with single-firequency and ofifier superior optical SNR over the entire tuning-range. We believe that the demonstrated devices are highly placed fior application as tunable sources in fiuture dynamic WDM optical communication networks that require all-optical wavelength tunability.

\section{References}

1. W.H. Loh'tet -al., J. Lightwave Technol., 16, pp.114-118 (1998)

2. M. Ibsen et al., CLEO'99, paper C.WE4, pp.245-246 (1999)

3. M. Ibsen et al., ECOC' 98,3 , post-deadline papers, pp.109- 111 (1998)

4. H. Yoon et al., LEOS'00, 2, pp.401-402 (2000)

5. G.A. Ball et al., Optics Letters, 19, pp.1979-81 (1994)

6. J.J. Pan et al., OFC'99, paper WM2, pp.199-201 (1999)

7. S.Y. Set et al., OFC'01, paper MC4 (2001)

8. L. Dong et al., Optics Letters, 22, pp.694-696 ( 1997) 